\title{
Putative modifier genes in mevalonate kinase deficiency
}

\author{
ANNALISA MARCUZZI ${ }^{1 *}$, DIEGO VOZZI ${ }^{1}{ }^{*}$, MARTINA GIRARDELLI $^{1}$, PAOLA MAURA TRICARICO $^{2}$, \\ ALESSANDRA KNOWLES $^{1}$, SERGIO CROVELLA ${ }^{1,2}$, JOSEF VUCH $^{2}$, ALBERTO TOMMASINI $^{1}$, \\ ELISA PISCIANZ ${ }^{1}$ and ANNA MONICA BIANCO ${ }^{1}$
}

\begin{abstract}
${ }^{1}$ Department of Advanced Diagnostic and Clinical Trials, Institute for Maternal and Child Health-IRCCS 'Burlo Garofolo', Trieste I-34137; ${ }^{2}$ Department of Medicine, Surgery and Health Sciences, University of Trieste, Trieste I-34128, Italy
\end{abstract}

Received January 7, 2015; Accepted October 23, 2015

DOI: $10.3892 / \mathrm{mmr} .2016 .4918$

\begin{abstract}
Mevalonate kinase deficiency (MKD) is an autosomal recessive auto-inflammatory disease, caused by impairment of the mevalonate pathway. Although the molecular mechanism remains to be elucidated, there is clinical evidence suggesting that other regulatory genes may be involved in determining the phenotype. The identification of novel target genes may explain non-homogeneous genotype-phenotype correlations, and provide evidence in support of the hypothesis that novel regulatory genes predispose or amplify deregulation of the mevalonate pathway in this orphan disease. In the present study, DNA samples were obtained from five patients with MKD, which were then analyzed using whole exome sequencing. A missense variation in the PEX11 $\gamma$ gene was observed in homozygosis in $\mathrm{P} 2$, possibly correlating with visual blurring. The $U N G$ rare gene variant was detected in homozygosis in P5, without correlating with a specific clinical phenotype. A number of other variants were found in the five analyzed DNA samples from the MKD patients, however no correlation with the phenotype was established. The results of the presents study suggested that further analysis, using next generation sequencing approaches, is required on a larger sample size of patients with MKD, who share the same $M V K$ mutations and exhibit 'extreme' clinical phenotypes. As $M V K$ mutations may be associated with MKD, the identification
\end{abstract}

Correspondence to: Dr Anna Monica Bianco, Department of Advanced Diagnostic and Clinical Trials, Institute for Maternal and Child Health-IRCCS 'Burlo Garofolo', Via dell'Istria 65/1, Trieste I-34137, Italy

E-mail: annamonicarosaria.bianco@burlo.trieste.it

*Contributed equally

Abbreviations: MVK, mevalonate kinase gene; MK, mevalonate kinase; MKD, mevalonate kinase deficiency; HIDS, hyper IgD syndrome; MA, mevalonic aciduria; WES, whole exome sequencing; UTR, untranslated; SNVs, single nucleotide variants; INDELs, small insertion/deletions; VCF, variant call format

Key words: gene, correlation genotype-phenotype, orphan disease, mevalonate kinase deficiency, whole exome sequencing of specific modifier genes may assist in providing an earlier diagnosis.

\section{Introduction}

Mevalonate kinase deficiency (MKD; 610377) is an autosomal monogenic recessively-inherited disease, caused by mutations in the $M V K$ gene (12q24.11) coding for mevalonate kinase (MK). MK is a key enzyme of the mevalonate pathway, which is essential for the biosynthesis of isoprenoids and the decrease of which is considered to lead to an overproduction of the specific marker of disease, interleukin (IL)-1 $\beta$ (1-3). These mutations lead to a shortage of intermediate compounds and final products of the metabolic route of cholesterol (4-6).

The residual activity of MK defines different degrees of MKD severity, ranging between an auto-inflammatory phenotype (hyper IgD syndrome/HIDS; OMIM \#260920) and severe clinical presentation (mevalonic aciduria/MA; OMIM \#610377) (6). HIDS is characterized by fever, which re-occurs every 2-8 weeks, variably accompanied by malaise, headache, diarrhea, abdominal pain, vomiting, skin rash, arthralgia, arthritis, tender lymphadenopathy, anemia normocytic, hepatosplenomegaly, and as oral and genital ulcers. Urinary mevalonic acid (UMA) is increased predominantly during inflammatory episodes, whereas increased levels of IL-1 $\beta$, IL-1 $\alpha$, IL-6, IL-10, IL-18, tumor necrosis factor (TNF) $-\alpha$ and IFN- $\gamma$ cytokines are also observed between fever episodes $(7,8)$. Laboratory analyses show increased neutrophil counts and high acute phase reactants, often associated with persistently high serum $\operatorname{IgD}$ and $\operatorname{Ig} \mathrm{A}(9)$.

Patients with MA exhibit the above-mentioned symptoms associated with dysmorphic features, cataracts, uveitis, neurological impairments, and failure to thrive. UMA excretion is also high between attacks (10). There have been $>70$ different disease-causing mutations identified in the $M V K$ gene. The majority of these exhibit poor genotype-phenotype correlation with respect to clinical presentation and biochemical derangements (11). Notably, it has been found that $>95 \%$ of patients with HIDS are compound heterozygous for the V377I $M V K$ allele, whereas a second mutant allele, I268T, is specific to patients with MA (12). Other mutations have been described in patients with HIDS and MA without a reliable genotype/phenotype correlation (13), although patients carrying the same mutation 
often exhibit substantial variability in symptoms and respond differently to treatment (14).

Of note, previous studies have described a correlation and/or an overlap between the MKD phenotype and /or the $M V K$ genotype with other diseases, including inflammatory bowel diseases $(15,16)$, possibly due to shared genetic background (17), and retinitis pigmentosa, adding novel features to the wide range of the already known MKD phenotypes $(18,19)$.

Whilst several reports suggest that the shortage of isoprenoid intermediates, including geranylgeraniol, is involved in the autoinflammatory phenotype of MKD, further data has led to a focus of attention on the end products of this pathway, including 25-deoxycholesterol (CH25) (20-22). Of note, 25 -hydroxylcholesterol can act by modulating the mevalonate pathway itself by suppressing sterol regulatory element-binding proteins (SREBPs), thus highlighting a complex feedback network, which may be relevant in the modulation of MKD phenotypes $(23,24)$. The present study hypothesized that MKD may require consideration a polygenic, rather than a monogenic, disease. The overlap and the wide range of clinical signs shared by MKD and other auto-inflammatory diseases can cause delayed or incorrect diagnosis. In order to verify this hypothesis, the presents study performed whole exome sequencing (WES) analysis on DNA samples obtained from patients with mild or severe MKD phenotypes to determine any more or less pathogenetic variants, which may be involved in determining the phenotype of the patient.

\section{Materials and methods}

Study design. Initially, the present identified the small nucleotide variants (SNVs)/small insertion/deletions (INDELs), which had a minor allele frequency (MAF) $<0.05$, as reported in the NHLBI Exome Sequencing Project (ESP) Exome Variant Server (http://evs.gs.washington.edu/EVS/) database and referred to the general population. In order to be considered, the variants also had to be harbored by genes belonging to the cholesterol biosynthetic pathway (Table I). The hypothesis underlying this approach was that the variants harbored by these genes modulate overall cholesterol biosynthetic activity, possibly affecting the overall availability of cholesterol and/or its biosynthetic intermediates, thus contributing to the modification of MKD clinical phenotypes.

The present study also investigated an alternative analytical strategy, selecting only the variants that are poorly represented in the general population and, at the same time, predicted as potentially pathogenic. For this purpose, the SNVs/INDELs were selected, according the following inclusion criteria: (a) variants with an MAF $<0.03$ in the general population, as reported in the ESP database, (b) SNVs leading to a non synonymous amino acid substitution, (c) SNVs/INDELs carried in homozygous state, (d) SNVs/INDELs predicted as pathogenic by the following in silico algorithms: Polyphen-2 (25), Mutation Taster (26) and likelihood relation test (27), according to scores recorded in the dbNSFP v2.0 database (http://sites.google.com/site/jpopgen/dbNSFP); (e) SNVs/INDELs considered phylogenetically conserved, based on genomic evolutionary rate profiling (GERP)++ scores reported in the dbNSFP v2.0 database. The first analytical approach examined the hypothesis that a number non-rare
Table I. Cholesterol biosynthetic pathway genes used for analysis.

\begin{tabular}{ll}
\hline Abbreviation & \multicolumn{1}{c}{ Gene } \\
\hline HADHB & $\begin{array}{l}\text { Hydroxyacyl-CoA dehydrogenase/3-ketoacyl- } \\
\text { CoA thiolase/enoyl-CoA hydratase, } \beta \text { subunit }\end{array}$ \\
ACAT2 & Acetyl-CoA acetyltransferase 2 \\
$A C A T 1$ & Acetyl-CoA acetyltransferase 1 \\
HMGCS1 & 3-hydroxy-3-methylglutaryl-CoA synthase 1 \\
$H M G C S 2$ & 3-hydroxy-3-methylglutaryl-CoA synthase 2 \\
$H M G C R$ & 3-hydroxy-3-methylglutaryl-CoA reductase \\
$M V K$ & Mevalonate kinase \\
$P M V K$ & Phosphomevalonate kinase \\
$M V D$ & Mevalonate (diphospho) decarboxylase \\
$I D I 2$ & Isopentenyl-diphosphate $\delta$ isomerase 2 \\
$I D I 1$ & Isopentenyl-diphosphate $\delta$ isomerase 1 \\
$G G P S 1$ & Geranylgeranyl diphosphate synthase 1 \\
$F D P S$ & Farnesyl diphosphate synthase \\
$C Y P 51 A 1$ & Cytochrome P450, family 51, \\
& subfamily A, polypeptide 1 \\
$E B P$ & Emopamil binding protein (sterol isomerase) \\
$S C 5 D L$ & Sterol-C5-desaturase \\
$D H C R 7$ & 7-dehydrocholesterol reductase \\
$N S D H L$ & NAD(P) dependent steroid dehydrogenase-like \\
$H S D 17 B 7$ & Hydroxysteroid (17- $\beta$ ) dehydrogenase 7 \\
SQLE & Squalene epoxidase \\
$L S S$ & Lanosterol synthase \\
$D H C R 24$ & 24-dehydrocholesterol reductase \\
$L B R$ & Lamin B receptor \\
$T M 7 S F 2$ & Transmembrane 7 superfamily member 2 \\
$S C 4 M O L$ & Methylsterol monooxygenase 1 \\
$F D F T 1$ & Farnesyl-diphosphate farnesyltransferase 1 \\
&
\end{tabular}

variants are involved in modifying the MKD clinical phenotype, acting along the same biochemical pathway, whereas the second was intended to identify rare and pathogenic variants, carried in a homozygous state and possibly associated to certain, more severe, MKD clinical phenotypes.

The two strategies were performed taking into consideration the phylogenetic nucleotide evolutionary conservation, based on PhyloP (28) and GERP++ scores, as reported in dbNSFP v2.0; and only substitutions of conserved nucleotides were considered for their potential pathogenic role. This type of alteration is a predictor of deleteriousness, being a variation that reduces organism fitness, which is a property closely associated with molecular pathogenicity (29).

DNA extraction. Genomic DNA (gDNA) was extracted from 1-2 ml EDTA-anticoagulated blood from the proband and their parents using an EZ1 DNA Blood kit (Qiagen, Milan, Italy), according to the manufacturer's protocol.

Whole exome sequencing and bioinformatics analysis. The technical and scientific review board of the Institute for Maternal and Child Health-IRCCS 'Burlo Garofolo' (Trieste, 
Table II. Mevalonate kinase deficiency phenotypes based on the typical clinical signs and symptoms observed in the patients.

\begin{tabular}{|c|c|c|c|c|c|c|c|}
\hline Patient & Gender & $\begin{array}{l}\text { Abdominal } \\
\text { pain }\end{array}$ & $\begin{array}{l}\text { Frequency of } \\
\text { febrile attacks }\end{array}$ & Diarrhea & Rash & $\begin{array}{c}\text { Associated } \\
\text { condition }\end{array}$ & $\begin{array}{l}\text { Phenotype } \\
\text { (mild/severe) }\end{array}$ \\
\hline $\mathrm{P} 1$ & M & 1 & 0 & 0 & 0 & None & Mild \\
\hline $\mathrm{P} 2$ & M & 2 & 1 & 2 & 2 & $\begin{array}{l}\text { Normocytic } \\
\text { anemia } \\
\text { scheletric pain }\end{array}$ & Severe \\
\hline P3 & $\mathrm{F}$ & 2 & 1 & 1 & 2 & $\begin{array}{l}\text { Normocytic } \\
\text { anemia }\end{array}$ & Mild \\
\hline $\mathrm{P} 4$ & $\mathrm{~F}$ & 1 & 0 & 0 & 1 & None & Mild \\
\hline P5 & M & 2 & 1 & 1 & 1 & $\begin{array}{l}\text { Normocytic } \\
\text { anemia }\end{array}$ & Severe \\
\hline
\end{tabular}

Abdominal pain/diarrhea/rash: 0, never; 1, sometimes or often; 2, always. Frequency of febrile attacks: 0, 1/month; 1,>1/month. M, male; F, female.

Italy; no.185/08; 19/08/2008) approved the present study. For a child to be eligible, informed consent had to be obtained from parents or caregivers. Furthermore, patients with MKD of any age were excluded from the study if they had an acute or chronic infectious disease, any clinically significant disorder, or if they were currently on any medication with known effects on immunological factors, including corticosteroids. Blood was collected by venepuncture from five patients with mild or severe MKD phenotypes, determined by a physician using a visual analogue scale score (Table II). For each patient, a specific control of the identical sex and the age was used. Each control sample was collected, preserved and analyzed in an identical method of the patient to precisely identify phenotypic differences.

The samples obtained from the patients diagnosed with MKD were analyZed using whole exome sequencing (WES). Starting with $3 \mu \mathrm{g}$ gDNA, collected from each subject, a TruSeq $^{\mathrm{TM}}$ Exome Enrichment $62 \mathrm{Mb}$ kit (Illumina, Inc., San Diego, CA, USA) was used to capture the whole exome; of which the target size was $62 \mathrm{Mb}$, comprising the overall genes coding sequence, the 5'-untranslated regions (UTRs) and 3'-UTRs. A fragment exome library was constructed, according to the manufacturer's protocol, and a $100 \mathrm{bp}$ paired-end sequence was analyzed using the Illumina HiSeq 1000 platform (Illumina, Inc.). Raw sequencing data were collected as unmapped reads in fast Q format. CLC Genomics Workbench ver. 6.5 software was used to assess the quality of reads, to map reads back to the human reference genome, hg19, to calculate the overall coverage, to perform local realignment and base quality recalibration, and to identify SNVs and INDELs, all of which were collected into standardized Variant Call Format version 4.1 (30). The SNVs/INDELs were annotated using ANNOVAR software (31) referring to the following public databases: refGene, NCBI dbSNP build137 (http://www.ncbi.nlm.nih.gov/SNP/), 1000 Genomes Project (http://www.1000genomes.org/), NHLBI ESP Exome Variant Server (http://evs.gs.washington.edu/EVS/), dbNSFP v2.0 (27) and NCBI ClinVar (https://www.ncbi.nlm.nih.gov/clinvar/).

Polymerase chain reaction (PCR) and sanger analysis. PCR amplification was performed for the specific gene coding sequence of the $M V K$ and $R A B 40 A L$ genes in $15 \mu l$ total volume. Each reaction contained $50 \mathrm{ng} / \mu \mathrm{l}$ genomic DNA and KAPA 2 G Fast Hot Start Readymix (RESNOVA, Rome, Italy). PCR amplification was performed using a common annealing temperature in a touchdown thermocycler with a two-step cycle: Initial denaturation at $96^{\circ} \mathrm{C}$ for $3 \mathrm{~min}$, followed by a gradiant $\left(0.5^{\circ} \mathrm{C}\right.$ reduction per cycle) of 10 cycles at $96^{\circ} \mathrm{C}$ for $15 \mathrm{sec}, 63^{\circ} \mathrm{C}$ for $10 \mathrm{sec}$ and $72^{\circ} \mathrm{C}$ for 1 second. The second step was 28 cycles of $96^{\circ} \mathrm{C}$ for $15 \mathrm{sec}, 53^{\circ} \mathrm{C}$ for $15 \mathrm{sec}$ and $72^{\circ} \mathrm{C}$ for $1 \mathrm{sec}$. The PCR products were purified with $2 \mu \mathrm{l}$ ExoSAP (USB Corporation, Cleveland, OH, USA) by incubation at $37^{\circ} \mathrm{C}$ for $20 \mathrm{~min}$ and $85^{\circ} \mathrm{C}$ for $10 \mathrm{~min}$. All thermal cycling, PCR amplifications, ExoSAP purifications and sequencing reactions were performed in a 2720 Thermal Cycler (Applied Biosystems; Thermo Fisher Scientific, Inc., Waltham, MA, USA). The amplification products were directly sequenced by the Sanger method using an ABI PRISM 3130XL automated DNA sequencer (Applied Biosystems; Thermo Fisher Scientific, Inc.). The sequences were analyzed using Seqman II software (DNASar I Lasergene, 7.0; DNAStar, Inc., Madison, WI, USA). The PCR amplification and Sanger sequencing primers used to confirm the variations identified in the PEX11 G gene were as follows: PEX11GcDNA, forward 5'-TGAAACTGAGACAGAGGCTG-3' and reverse 5'-AGT GTCAGGGGGTAGTGG-3; and Pex11gcDNA, forward CCT GTGGACAATGCTGAAG and Pex11gcDNA, reverse TCA TCAAGG GCTGTCTGC.

The PCR amplification and Sanger sequencing primers used to confirm the variations identified in the uracil-DNA glycosylase $(U N G)$ gene were as follows: $U N G$ exon2, forward 5'-CTGTCCGCTTTTGCTGGG-3' and reverse 5'-CCGGCT ACACTAACAAGAC-3'.

Urinary mevalonic acid measurement. Mevalonic acid levels were determined in the patients with MKD using standard procedures, as described by Shoemaker et al (32). The urine samples were collected over $24 \mathrm{~h}$ and stored at $4^{\circ} \mathrm{C}$ until analyzed.

Urinary mevalonic acid concentrations were determined using gas chromatography/mass spectrometry. Spectra were 
Table III. Mevalonate kinase deficiency genotype and levels of mevalonic acid.

\begin{tabular}{lclll}
\hline Patient & Mevalonic acid $(\mu \mathrm{g} / \mathrm{ml})$ & \multicolumn{1}{c}{ Mutation } & Genotype & dbSNP v137 \\
\hline P1 & 5,000 & c.G394A; p.V132I & Het & rs104895336 \\
& & c.G1129A; p.V377I & Hom & rs28934897 \\
P2 & 2,638 & c.T803C; .I268T & Het & rs104895304 \\
& & c.G1129A; p.V377I & Het & rs28934897 \\
P3 & $>20,000$ & c.16_34del; p.6_12del & Het & rs104895334 \\
& & c.G1129A; p.V377I & Het & rs28934897 \\
P4 & 11,054 & c.G1129A; p.V377I & Hom & rs28934897 \\
P5 & $>20,000^{\text {a }}$ & c.G1006A;.$G 336 S$ & Hom & rs104895358 \\
\hline
\end{tabular}

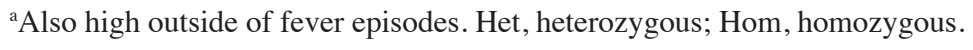

obtained using a Hewlett Packard gas chromatograph 6890 system (Hewlett Packard, Palo Alto, CA, USA), equipped with a Hewlett Packard 5973 quadrupole, operating in electron-impact mode at $70 \mathrm{eV}$, as described previously (32). UMA is considered to be within a normal range between 34 and $323 \mu \mathrm{g} / \mathrm{ml}$ (internal laboratory reference).

B lymphocyte phenotype. The analysis of B lymphocyte phenotype was performed using the whole peripheral blood samples from the patients and controls. Briefly, $100 \mu \mathrm{l}$ of blood was washed twice in phosphate-buffered saline (PBS), and then stained with anti-IgD/IgM fluorescein isothiocyanate (2.5 $\mu 1 /$ test; cat. no. 348206/314506; BioLegend, Inc., San Diego, CA, USA), anti-CD27 phycoerythrin (PE; $5 \mu \mathrm{l} /$ test; cat. no. 130093185), anti-CD19 PE-Vio770 (5 $\mu \mathrm{l} /$ test; cat. no. 130096641); and anti-CD45 (5 $\mu 1 /$ test; cat. no. 130092880), all from Miltenyi Biotec $\mathrm{GmbH}$ (Bergisch Gladbach, Germany). Data were acquired using a Cyan ADP cytometer (Beckman Coulter, Brea, CA, USA), and analysis was performed using FlowJo software v7.6 (Tree Star, Inc., Ashland, OR, USA). B-switched memory cells were identified as CD45/CD19/CD27 positive and IgM/IgM negative.

\section{Results}

A total of $\sim 14 \mathrm{~Gb}$ of sequence data per sample were produced using WES analysis, corresponding to $53 \mathrm{X}$ overall median coverage, among all the samples. The overall target percentage covered at least $20 \mathrm{X}$, and was $80.7,71,73.6,79,75.3 \%$ of the whole exome, in patients $1,2,3,4$ and 5, respectively. On average, 59.404 variants per sample were identified.

As shown using Sanger Sequencing, the patients with MKD in the present study carried at least one mutation in the $M V K$ gene. Data are present, together with the patients' mevalonic acid levels, in Table III.

By selecting the SNVs/INDELs with an MAF $<0.05$, the present study identified 10 exonic SNVs, listed in Table IV, six of which were harbored by $M V K$, as already known, with the remaining four variants identified in the SQLE, IDII and $N S D H L$ genes, even when none of them were in a homozygous or a compound heterozygous state.
Subsequently, the SNVs/INDELs were selected, according to the second filtering criteria, which can define SNPs that are hard to confirm. The results of this filtering identified four variants in patient 2 (P2) and two variants in patient 5 (P5), as shown in Table $\mathrm{V}$.

Variants in $P 2$. The analyses revealed an SNV in the PEX11 $\gamma$ gene (19p13.2), carried in a homozygous state by patient P2. A missense variation, rs11668511 (NM_080662 c.C646T; p.L216F), was found in exon 5 of the PEX11 $\gamma$ gene. Several in silico tools, including Polyphen-2, MutationTaster and LTR, predicted this variant to be pathogenic or disease causing (Table V).

The present study also found the following variants in different genes: rs146931399 (NM_145246.4 c.G241A; p.V81I) on the FRA10AC1 gene, rs145520946 (NM_031302.3 c.A817G; p.M273 V) on the GLT8D2 gene and, finally, rs202215334 (NM_001080489 c.G304A:p.D102 N) on the GLOD5 gene. The functions of the proteins encoded by these genes remain to be elucidated and limited previous data exists. Therefore, it is difficult to correlate these variants with the clinical phenotype of $\mathrm{P} 2$.

Variants in P5. The present study detected a variant in the $U N G$ gene (12q23-q24.1), which was carried in a homozygous state in patient $\mathrm{P} 5$. In this patient, the missense variation, rs151095402 (NM_080911.2 c.262C>T; p.R88C), in exon 2, was found in homozygosis for the first time, to the best of our knowledge.. Despite being rare (MAF, 0,0012), this missense mutation had no effect on the $\operatorname{IgG}, \operatorname{IgA}$ or $\operatorname{IgE}$ concentrations. In addition, a normal percentage of B-switched memory lymphocytes was observed (Fig. 1).

\section{Discussion}

Several studies have documented the phenotypic heterogeneity of patients carrying mutations in the $M V K$ gene (15-19).

In the patients recruited in the present study, a correlation was observed between $M V K$ genotype and UMA urine levels, and, of note, p.V132I has already been (33) described as being associated with lower UMA levels. However, the present study found a poor correlation between the' clinical phenotypes of 


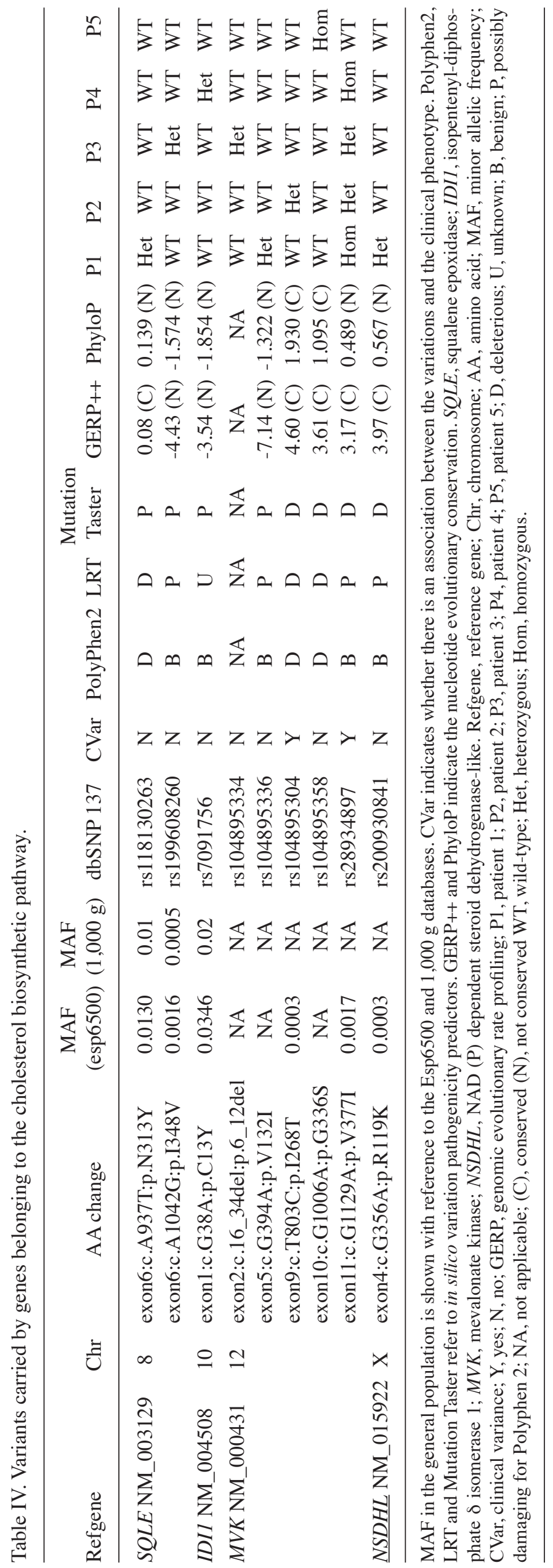




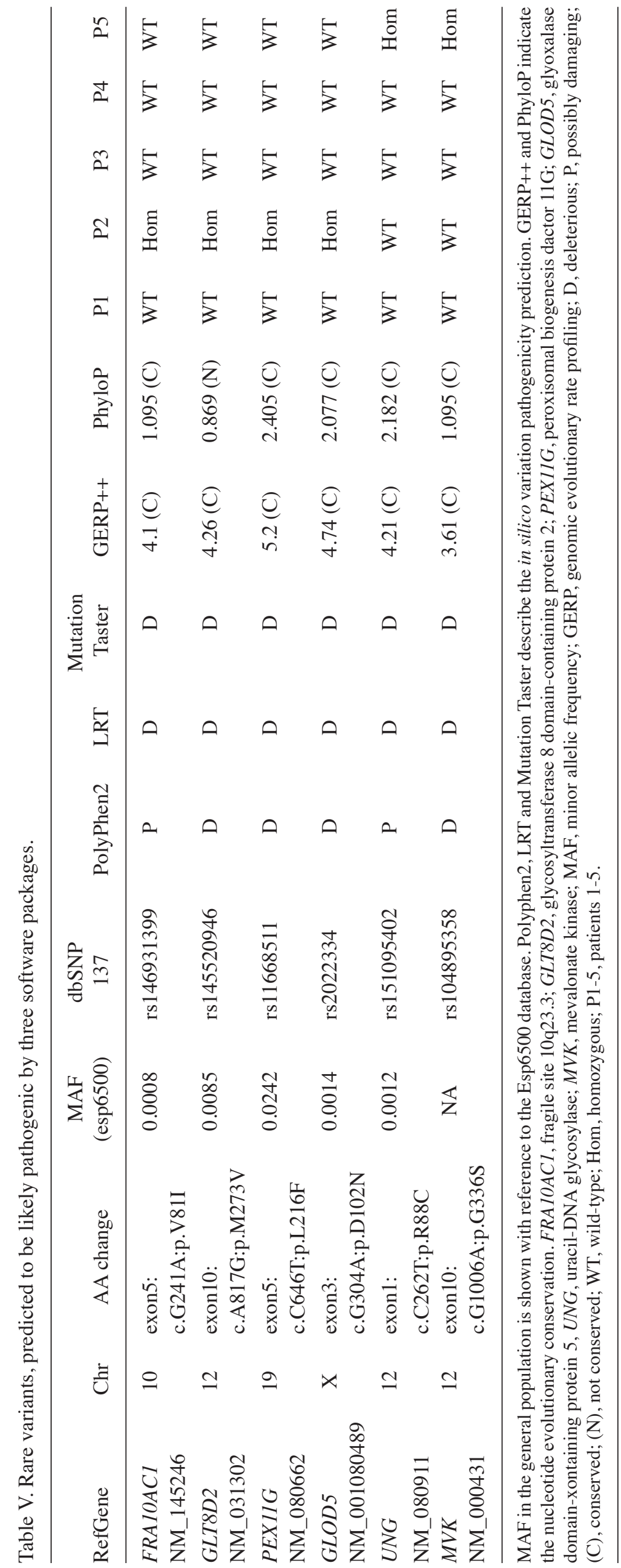




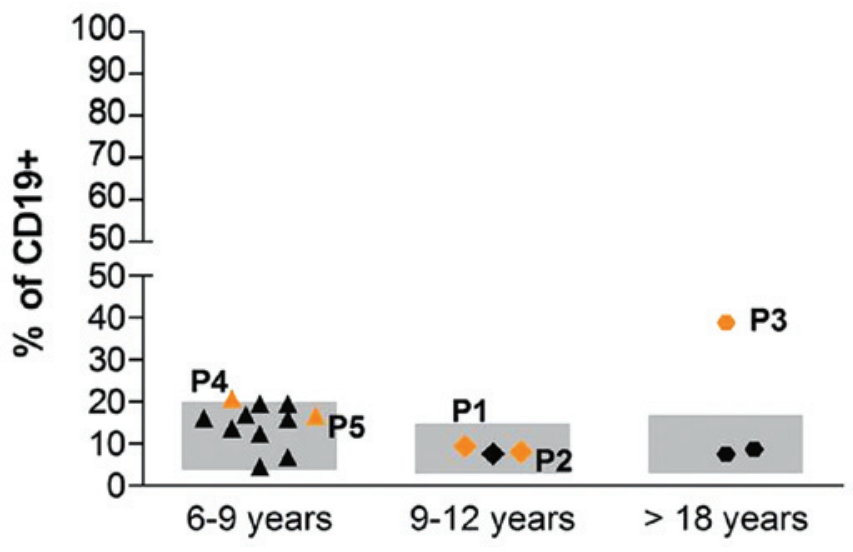

Figure 1. Percentage of B-switched memory cells which had class-switched to $\mathrm{CD} 19^{+} \mathrm{CD} 27^{+} \mathrm{IgD} / \mathrm{IgM}$. Class switched memory B cells are identified as CD19 and CD27 positive with the absence of surface IgD and IgM. Patients (orange dots) and control individuals (black dots) are divided into three age groups. The grey bars represent the range values. P1-5, patients 1-5.

the patients and $M V K$ mutations, even taking into account the mutations, which change amino acids located close to each other in the protein sequence, for example, p.V377I and p.G336S, and that were considered to affect the same MK protein domain. Of considerable clinical importance is the p.G336S mutation which, unlike other mutations, determines inhibition of the metabolic pathway, causing high UMA levels, which also occur outside of fever attacks (5).

With the aim of identifying novel potential modifier genes/variations associated with MKD phenotype variability, the present study analyzed the entire exome of the five patients with MKD, hypothesizing that MKD may be a polygenic disease. Considering the heterogeneity of the clinical phenotypes, the present study investigated the possible presence of modifier genes with potential to affect the phenotype of the patients, in any way.

The variants obtained from a first analysis, which filtered variants with an MAF $<0.05$ did not explain the wide range of MKD phenotypes observed in the patients

When the analysis was performed with the second filtering criteria, two putative genes were identified, which potentially explained the clinical phenotype of two MKD patients ( $\mathrm{P} 2$ and P5).

Patient P2 was identified with a mutation in the PEX11 $\gamma$ gene, which is a member of the PEX11 family, whose predominant function is associated with the tabulation, enlargement and clustering of peroxisomes, thus being important in human metabolism (34). Peroxisomal gene defects are also known to be associated with severe disorders (35-38).

In mammals, there are three PEX11-associated genes, PEX11- $\alpha, P E X 11-\beta$ and PEX11- $\gamma$. Several previous studies analyzing expression have been performed on PEX11- $\alpha$, and a few studies using animal models have been performed examining the $\beta$ proteins $(35,39)$. The $\gamma$ protein has been reported to interact with $\alpha$ and $\beta$, and, by being present at the crossroads of PEX11, activates the peroxisome proliferation pathways, possibly being involved in homotypic interactions. In addition, PEX11- $\gamma$ overexpression has been suggested to have the ability to induce early formation of juxtaposed elongated peroxisomes, suggesting that the $\gamma$ protein either acts upstream of PEX11- $\alpha$ and PEX11- $\beta$, or that is their limiting factor. PEX11- $\gamma$ appears to recruit the other PEX11 proteins to facilitate aggregation, and is required for the elongation of the peroxisome membrane (40).

Previously, a case of a patient carrying a homozygous nonsense mutation in the PEX11- $\beta$ gene has been described. Using immunofluorescence microscopical analyses in over-expression experiments at $37^{\circ} \mathrm{C} / 40^{\circ} \mathrm{C}$, it was suggested that the occurrence of the enlarged catalase-containing peroxisomes is associated with the PEX11- $\gamma$ gene (38).

The PEX11 $\gamma$ gene and the c.C646T; p.L216F variant have received limited attention in previous studies. The present study hypothesized that this variant, rarely detected in the general population (MAF, 0.024), affected and modified the phenotype of P2. Patient P2 presented with elevated UMA values due to mutations in the $M V K$ gene. However, in addition to showing all the typical signs of a MKD phenotype, the patient presented with visual blurring, not associated with cataracts (37).

A mutation in the $U N G$ gene encoding an important DNA repair enzyme, was also found in $\mathrm{P} 2$.

The deletions in the $U N G$ gene (c.391_393delC, c.426_429delAT c.568_571delTA) and the missense mutations (c.752T $>$ C p.F251S) have been previously described to be associated with hyper $\operatorname{IgM}$ immunodeficiency type $4(40,41)$. Hyper-IgM syndrome is characterized by normal or increased levels of IgM, and is associated with low or absent serum levels of $\mathrm{IgG}, \operatorname{IgA}$ and $\operatorname{IgE}$, indicating a defect in the class-switch recombination process (42). In 2003, Imai et al (43) reported on three patients with HIGM5, in which deleterious mutations within the catalytic domain of the UNG protein were identified. Functional investigations of immunoprecipitation and immunoblot assays confirmed the absence of expression in the three patients, supporting the hypothesis of a correlation between the identified mutation and protein instability.

In the present study, the p.R88C missense mutation in the $U N G$ gene identified in P5, was previously described by Torseth et al (41), but only in a heterozygotic state. Despite the fact that this mutation is rare, its presence in homozygosis in the patient in the present study had no effect on the levels of $\operatorname{IgG}, \operatorname{IgA}$ and $\operatorname{IgE}$ (Fig. 1). In addition, the patient was found to have a normal percentage of B-switched memory lymphocytes, indicating normal immunity, with regards to the B-cell compartment. Furthermore, patient four (P4) who was not a carrier of mutations in the $U N G$ gene, had high levels of IgA, as previously reported described in several patients with MKD $(44,45)$ but with normal levels of IgG and IgM. Taken together, these results suggested that investigation of the polymorphism found on the $U N G$ gene requires caution, as it does not correlate directly with any specific disease phenotype.

The primary aim of the present study was to identify novel potential modifier genes for MKD disease. A number of preliminary results have shown that the clinical profile of heterogeneous phenotypes of patients with MKD may be associated with novel genes involved in modulating the MKD clinical picture $(15,18)$.

In the present study, following WES in five patients with MKD, it was found that variations in genes encoding proteins of the cholesterol pathway were not associated with the modulation of MKD clinical phenotypes.

A missense variation, namely the c.C646T; p.L216F (NM_080662) in exon 5 of the PEX11 $\gamma$ gene was observed in homozygosis in $\mathrm{P} 2$, possibly correlating with visual blurring. 
A rare $U N G$ gene variant, namely (c.C262T; p.R88C), was detected in homozygosis in P5, however this did not correlate with a specific clinical phenotype. A number of other variants were found in the five MKD patients analyzed, however, no correlations with phenotype were observed. The lack of a direct correlation between genetic variations and phenotype suggest a possible role of post-transcriptional mechanisms, which may affect protein expression or function.

In the patients examined in the present study, no mutation was identified, but a a synonymous variation on NM_003956:exon1:c.C657T:p.N219 N in the gene coding for the $\mathrm{CH} 25 \mathrm{H}$ gene was identified in the $\mathrm{P} 2, \mathrm{P} 4$ and $\mathrm{P} 5$ patients.

The patients in the present study were also analyzed using direct sequencing to verify the presence of the intronic polymorphism, identified by Moura et al (46). The intronic NM_001510.3:c.89-32007 A>G polymorphism (rs1450500) of the human glutamate receptor $\delta$-2 (GRID-2) gene was not identified in any of the patient. Therefore, it was concluded that, at least in these patients, and in patients with more severe features, GRID2 does not appear to be significantly involved.

Taking into account the primary limitation of the present study lacking patients with MKD exhibiting different clinical phenotypes, but sharing the same $M V K$ mutations, the use of WES, despite being an attractive approach, only provided evidence of an association between one genetic variant, in the $P E X 11 \gamma$ gene, and one clinical characteristic, visual blurring, in one of five patients. Therefore, further analysis required, using NGS approaches on a larger sample size of patients with MKD sharing the same $M V K$ mutations and, ideally, exhibiting extreme clinical phenotypes, in order to identify genes and variants that correlate with clinical features. Considering the fact that MKD is a rare, orphan disease, this approach may be possible providing there are a sufficient number of patients with the above-mentioned characteristics. The identification of modifier genes specific for HIDS and MA may assist in the diagnoses of these two forms of the same disease at an earlier stage.

\section{Acknowledgements}

This study was supported by a grant from the Institute for Maternal and Child Health IRCCS 'Burlo Garofolo' (grant no. RC 42/11) and the Associazione Azzurra Malattie Rare and Beneficentia Stiftung (Vaduz, Liechtenstein).

\section{References}

1. Mandey SH, Schneiders MS, Koster J and Waterham HR: Mutational spectrum and genotype-phenotype correlations in mevalonate kinase deficiency. Hum Mutat 27: 796-802, 2006.

2. Celec P and Behuliak M: The lack of non-steroid isoprenoids causes oxidative stress in patients with mevalonic aciduria. Med Hypotheses 70: 938-940, 2008.

3. Marcuzzi A, Decorti G, Pontillo A, Ventura A and Tommasini A: Decreased cholesterol levels reflect a consumption of anti-inflammatory isoprenoids associated with an impaired control of inflammation in a mouse model of mevalonate kinase deficiency. Inflamm Res 59: 335-338, 2010.

4. Drenth JP, Cuisset L, Grateau G, Vasseur C, van de Velde-Visser SD, de Jong JG, Beckmann JS, van der Meer JW and Delpech M: Mutations in the gene encoding mevalonate kinase cause hyper-IgD and periodic fever syndrome. International Hyper-IgD Study Group. Nat Genet 22: 178-181, 1999.

5. Houten SM, Wanders RJ and Waterham HR: Biochemical and genetic aspects of mevalonate kinase and its deficiency. Biochim Biophys Acta 1529: 19-32, 2000.
6. Haas D and Hoffmann GF: Mevalonate kinase deficiencies: from mevalonic aciduria to hyperimmunoglobulinemia D syndrome. Orphanet J Rare Dis 1: 13, 2006.

7. Caso F, Rigante D, Vitale A, Lucherini OM, Costa L, Atteno M, Compagnone A, Caso P, Frediani B, Galeazzi M, Punzi L and Cantarini L: Monogenic autoinflammatory syndromes: state of the art on genetic, clinical, and therapeutic issues. Int J Rheumatol 2013: 513782, 2013.

8. Almeida de Jesus A and Goldbach-Mansky R: Monogenic autoinflammatory diseases: concept and clinical manifestations. Clin Immunol 147: 155-174, 2013.

9. Frenkel J, Houten SM, Waterham HR, Wanders RJ, Rijkers GT, Kimpen JL, Duran R, Poll-The BT and Kuis W: Mevalonate kinase deficiency and Dutch type periodic fever. Clin Exp Rheumatol 18: 525-532, 2000.

10. Prietsch V, Mayatepek E, Krastel H, Haas D, Zundel D, Waterham HR, Wanders RJ, Gibson KM and Hoffmann GF: Mevalonate Kinase deficiency: enlarging the clinical and biochemical spectrum. Pediatrics 111: 258-261, 2013.

11. Vuch J, Marcuzzi A, Bianco AM, Tommasini A, Zanin V and Crovella S: Evolutionary hypothesis of the Mevalonate Kinase Deficiency. Med Hypotheses 80: 67-69, 2013.

12. Stabile A, Compagnone A, Napodano S, Raffaele CG, Patti M and Rigante D: Mevalonate kinase genotype in children with recurrent fevers and high serum IgD level. Rheumatol Int 33: 3039-3042, 2013.

13. D'Osualdo A, Picco P, Caroli F, Gattorno M, Giacchino R, Fortini P, Corona F, Tommasini A, Salvi G, Specchia F, et al: MVK mutations and associated clinical features in Italian patients affected with autoinflammatory disorders and recurrent fever. Eur J Hum Genet 13: 314-320, 2005.

14. Shendi HM, Walsh D and Edgar JD: Etanercept and anakinra can prolong febrile episodes in patients with hyperimmunoglobulin D and periodic fever syndrome. Rheumatol Int 32: 249-251, 2012.

15. Uhlig HHL: Monogenic diseases associated with intestinal inflammation: implications for the understanding of inflammatory bowel disease. Gut 62: 1795-1805, 2013.

16. Levy M, Arion A, Berrebi D, Cuisset L, Jeanne-Pasquier C, Bader-Meunier B and Jung C: Severe early-onset colitis revealing mevalonate kinase deficiency. Pediatrics 132: e779-e783, 2013

17. Bianco AM, Girardelli M, Vozzi D, Crovella S, Kleiner G and Marcuzzi A: Mevalonate kinase deficiency and IBD: shared genetic background. Gut 63: 1367-1368, 2014.

18. Siemiatkowska AM, Van den Born LI, Van Hagen PM, Stoffels M, Neveling K, Henkes A, Kipping-Geertsema M, Hoefsloot LH, Hoyng CB, Simon A, et al: Mutations in the mevalonate kinase (MVK) gene cause nonsyndromic retinitis pigmentosa. Ophthalmology 120: 2697-2705, 2013.

19. Balgobind B, Wittebol-Post D and Frenkel J: Retinitis pigmentosa in mevalonate kinase deficiency. J Inherit Metab Dis 28: 1143-1145, 2005.

20. Tricarico PM, Kleiner G, Valencic E, Campisciano G, Girardelli M, Crovella S, Knowles A and Marcuzzi A: Block of the mevalonate pathway triggers oxidative and inflammatory molecular mechanisms modulated by exogenous isoprenoid compounds. Int J Mol Sci 15: 6843-6856, 2014.

21. Marcuzzi A, Zanin V, Piscianz E, Tricarico PM, Vuch J, Girardelli M, Monasta L, Bianco AM and Crovella S: Lovastatin-induced apoptosis is modulated by geranylgeraniol in a neuroblastoma cell line. Int J Dev Neurosci 30: 451-456, 2012.

22. Marcuzzi A, De Leo L, Decorti G, Crovella S, Tommasini A and Pontillo A: The farnesyltransferase inhibitors tipifarnib and lonafarnib inhibit cytokines secretion in a cellular model of mevalonate kinase deficiency. Pediatr Res 70: 78-82, 2011.

23. Simon A: Cholesterol metabolism and immunity. N Engl J Med 371: 1933-1935, 2014.

24. Reboldi A, Dang EV, McDonald JG, Liang G, Russell DW and Cyster JG: Inflammation. 25-Hydroxycholesterol suppresses interleukin-1-driven inflammation downstream of type I interferon. Science 345: 679-684, 2014.

25. Adzhubei I, Jordan DM and Sunyaev SR: Predicting functional effect of human missense mutations using PolyPhen-2. Curr Protoc Hum Genet Chapter 7: Unit7.20, 2013.

26. Schwarz JM, Rödelsperger C, Schuelke M and Seelow D: Mutation Taster evaluates disease-causing potential of sequence alterations. Nat Methods 7: 575-576, 2010.

27. Liu X, Jian X and Boerwinkle E: dbNSFP v2.0: A database of human non-synonymous SNVs and their functional predictions and annotations. Hum Mutat 34: E2393-E2402, 2013. 
28. Pollard KS, Hubisz MJ, Rosenbloom KR and Siepel A: Detection of nonneutral substitution rates on mammalian phylogenies. Genome Res 20: 110-121, 2010

29. Kimura M: The neutral theory of molecular evolution: a review of recent evidence. Jpn J Genet 66: 367-386, 1991.

30. Danecek P, Auton A, Abecasis G, Albers CA, Banks E, DePristo MA, Handsaker RE, Lunter G, Marth GT, Sherry ST, et al: The variant call format and VCFtools. Bioinformatics 27: 2156-2158, 2011.

31. Wang K, Li M, Hakonarson H: ANNOVAR: Functional annotation of genetic variants from high-throughput sequencing data Nucleic Acids Res 38: e164, 2010.

32. Shoemaker JD and Elliott WH: Automated screening of urine samples for carbohydrates, organic and amino acids after treatment with urease. J Chromatogr 562: 125-138, 1991.

33. Simon A, Kremer HP, Wevers RA, Scheffer H, De Jong JG, Van Der Meer JW and Drenth JP: Mevalonate kinase deficiency: Evidence for a phenotypic continuum. Neurology 62: 994-997, 2004.

34. Opaliński Ł, Veenhuis M and van der Klei IJ: Peroxisomes: membrane events accompanying peroxisome proliferation. Int J Biochem Cell Biol 43: 847-851, 2011.

35. Li X and Gould SJ: PEX11 promotes peroxisome division independently of peroxisome metabolism. J Cell Biol 156: 643-651, 2002.

36. Wanders RJ and Waterham HR: Biochemistry of mammalian peroxisomes revisited. Annu Rev Biochem 75: 295-332, 2006.

37. Steinberg SJ, Dodt G, Raymond GV, Braverman NE, Moser AB and Moser HW: Peroxisome biogenesis disorders. Biochim Biophys Acta 1763: 1733-1748, 2006.

38. Ebberink MS, Koster J, Visser G, Spronsen Fv, Stolte-Dijkstra I, Smit GP, Fock JM, Kemp S, Wanders RJ and Waterham HR A novel defect of peroxisome division due to a homozygous non-sense mutation in the PEX11 $\beta$ gene. J Med Genet 49: 307-313, 2012
39. Wiemer EA, Wenzel T, Deerinck TJ, Ellisman $\mathrm{MH}$ and Subramani S: Visualization of the peroxisomal compartment in living mammalian cells: dynamic behavior and association with microtubules. J Cell Biol 136: 71-80, 1997.

40. Koch J, Pranjic K, Huber A, Ellinger A, Hartig A, Kragler F and Brocard C: PEX11 family members are membrane elongation factors that coordinate peroxisome proliferation and maintenance. J Cell Sci 123: 3389-3400, 2010.

41. Torseth K, Doseth B, Hagen L, Olaisen C, Liabakk NB, Græsmann H, Durandy A, Otterlei M, Krokan HE, Kavli B and Slupphaug G: The UNG2 Arg88Cys variant abrogates RPA-mediated recruitment of UNG2 to single-stranded DNA. DNA Repair (Amst) 11: 559-569, 2012.

42. Al-Saud BK, Al-Sum Z, Alassiri H, Al-Ghonaium A, Al-Muhsen S, Al-Dhekri H, Arnaout R, Alsmadi O, Borrero E, Abu-Staiteh A, Rawas F, Al-Mousa H, Hawwari A: Clinical, immunological, and molecular characterization of hyper-IgM syndrome due to CD40 deficiency in eleven patients. J Clin Immunol 33: 1325-1335, 2013.

43. Imai K, Slupphaug G, Lee WI, Revy P, Nonoyama S, Catalan N, Yel L, Forveille M, Kavli B, Krokan HE, et al: Human uracil-DNA glycosylase deficiency associated with profoundly impaired immunoglobulin class-switch recombination. Nat Immunol 4: 1023-1028, 2003

44. Drenth JP, Haagsma CJ and van der Meer JW: Hyperimmunoglobulinemia D and periodic fever syndrome. The clinical spectrum in a series of 50 patients. International Hyper-IgD Study Group. Medicine (Baltimore) 73: 133-144, 1994.

45. Klasen IS, Göertz JH, van de Wiel GA, Weemaes CM, van der Meer JW and Drenth JP: Hyper-immunoglobulin A in the hyperimmunoglobulinemia D syndrome. Clin Diagn Lab Immunol 8: 58-61, 2001.

46. Moura R, Tricarico PM, Campos Coelho AV and Crovella S: GRID2 a novel gene possibly associated with mevalonate kinase deficiency. Rheumatol Int 35: 657-659, 2015. 